Article

\title{
Pandoraea sp. RB-44, A Novel Quorum Sensing Soil Bacterium
}

\author{
Robson Ee Han-Jen, Yin Wai-Fong and Chan Kok-Gan* \\ Division of Genetics and Molecular Biology, Institute of Biological Sciences, Faculty of Science, \\ University of Malaya, 50603 Kuala Lumpur, Malaysia; E-Mails: robsonee@live.com (R.E.H.-J.); \\ yinwaifong@yahoo.com (Y.W.-F.)
}

* Author to whom correspondence should be addressed; E-Mail: kokgan@um.edu.my;

Tel.: +60-37967-5162; Fax: +60-37967-4509.

Received: 26 August 2013; in revised form: 20 September 2013 / Accepted: 30 September 2013/ Published: 18 October 2013

\begin{abstract}
Proteobacteria are known to communicate via signaling molecules and this process is known as quorum sensing. The most commonly studied quorum sensing molecules are $\mathrm{N}$-acylhomoserine lactones (AHLs) that consists of a homoserine lactone moiety and an $\mathrm{N}$-acyl side chain with various chain lengths and degrees of saturation at the C-3 position. We have isolated a bacterium, RB-44, from a site which was formally a landfill dumping ground. Using matrix-assisted laser desorption ionization time-of-flight (MALDI-TOF) mass spectrometry analysis, this isolate was identified as a Pandoraea sp.which was then screened for AHL production using biosensors which indicated its quorum sensing properties. To identify the AHL profile of Pandoraea sp. RB-44, we used high resolution tandem mass spectrometry confirming that this isolate produced $N$-octanoylhomoserine lactone (C8-HSL). To the best of our knowledge, this is the first report that showed quorum sensing activity exhibited by Pandoraea sp. Our data add Pandoraea sp. to the growing number of bacteria that possess QS systems.
\end{abstract}

Keywords: Pandoraea sp.; matrix-assisted laser desorption ionization time-of-flight (MALDI-TOF); mass spectrometry; triple quodruopole liquid chromatography mass spectrometry; $N$-octanoylhomoserine lactone (C8-HSL); quorum sensing 


\section{Introduction}

Initially often reported as non-fermentative Gram-negative bacilli or misidentified as Burkholderia cepacia complex (Bcc) or Ralstonia spp. in clinical microbiology laboratory [1-3], Pandoraea sp. is actually a taxonomically distinct $\beta$-subclass of proteobacteria where genera of Bukholderia and Ralstonia are the closest neighbors [4]. As an opportunistic pathogen with an unclear role in clinical colonization, Pandoraea spp. have been isolated from various patients, mainly with cystic fibrosis [5-8]. Pandoraea spp. are also well known for their bio-catalytic activities in a variety of reactions such as carbapenem hydrolysis [9] polychlorinated biphenyls (PCBs) biodegradation [10], sulphur oxidation [11] and lignin degradation [12]. Despite all the documentation on this species, little is known about the mechanism and regulation of its pathogenicity and bio-catalytic activities.

Most Gram negative bacteria use quorum sensing (QS) to establish communications between neighboring cells through secretion and detection of diffusible signaling molecule known as autoinducers [13-15]. QS is important as it regulates diverse bacterial physiological activities, such as swimming and twitching motility, bioluminescence, swarming, stimulate production of virulence in flora and fauna pathogens, biofilm differentiation, antibiotic biosynthesis, plasmid conjugal transfer and many more activities $[13,16,17]$. By far, the most widely studied QS molecules are $N$-acyl-homoserine lactones (AHLs) which are synthesized by AHL synthase (luxI homologue) that will diffuse and bind to its cognate receptor (luxR homologue) where this AHL-LuxR complex will be activated to regulate QS-mediated genes expression [18,19]. Thus, the best way to understand the molecular basis of Pandoraea sp. virulence factor and bio-catalytic activities is through characterizing its AHL production expression [20].

Here, we present a strain of Pandoraea sp. RB-44 isolated through four cycles of enrichment in KGm medium [21] from an ex-landfill site that activates both C. violaeum CV026 and E. coli [pSB401] biosensors. Subsequently, we used matrix-assisted laser desorption ionization time-of-flight (MALDI-TOF) mass spectrometry analysis for strain identification and high resolution quadrupole mass spectrometry to confirm its AHL profile. To our knowledge, this is the first report of AHL production by Pandoraea sp.

\section{Experimental Section}

\subsection{Soil Sampling}

Soil sampling was conducted in 2012 from an ex-landfill site in Ayer Hitam, Puchong (Malaysia) with the GPS coordinates of N03'00'12.1, E101'39'33'1 at an elevation of $61 \mathrm{~m}$ above sea level. The soil sample was collected from the soil surface at a depth of $10 \mathrm{~cm}$ and soil sample was placed into a sterile 50mL plastic tube. Upon arrival in the laboratory, the soil sample was processed with removal of large particulate organic matter using a sterile spatula.

\subsection{Enrichment and Isolation}

Enrichment and isolation of bacteria was done according to a previously described method [22] with slight modifications. Briefly, a soil sample (1 g) was resuspended in KGm medium (10 mL). The 
mixture was vigorously vortexed and the soil suspension was transferred (10\% v/v) into fresh KGm medium supplemented with 3-oxo-C6-HSL (50 mM final concentration, Sigma-Aldrich, St Loius, MO, USA) as the sole carbon sources. The mixture was incubated in $28{ }^{\circ} \mathrm{C}$ with shaking (220 rpm) for $48 \mathrm{~h}$. Similar transfers were repeated three times. At the fourth enrichment cycle, a diluted suspension was plated on Luria-Bertani (LB) agar and a plate of 3-oxo-C6-HSL-containing KGm agar to isolate pure colonies.

\subsection{Bacteria Strains and Culture Conditions}

All strains (Table 1) were routinely grown aerobically in LB medium with shaking (220 rpm) and LB agar at $28{ }^{\circ} \mathrm{C}$ unless otherwise stated. The composition of LB medium was: tryptone $(10 \mathrm{~g} / \mathrm{L})$, yeast extract $(5 \mathrm{~g} / \mathrm{L})$ and sodium chloride $(10 \mathrm{~g} / \mathrm{L})$ with additional bacto-agar $(15 \mathrm{~g} / \mathrm{L})$ in $\mathrm{LB}$ agar. For AHL extraction, bacteria strains were grown in a modified LBmedium buffered with $50 \mathrm{mM}$ 3-[N-morpholino] propanesulfonic acid (MOPS) to $\mathrm{pH} 5.5$ to prevent alkaline hydrolysis of any AHLs.

Table 1. Strains used in this study.

\begin{tabular}{|c|c|c|}
\hline Strain & Description & Source/Reference \\
\hline $\begin{array}{l}\text { Chromobacterium violaceum } \\
\text { CV026 }\end{array}$ & $\begin{array}{l}\text { Double mini-Tn5 mutant derived } \\
\text { from ATCC31532. Biosensor } \\
\text { that synthesize purple violacein } \\
\text { pigment in response of short } \\
\text { chain exogenous AHL. } \\
\text { Positive control for QS } \\
\text { properties. Capable of producing }\end{array}$ & [23] \\
\hline Erwinia carotovora GS101 & $\begin{array}{l}\text { AHL to activate C. violaeum } \\
\text { CV026. }\end{array}$ & Gift from Prof. Paul Williams \\
\hline Erwinia carotovora PNP22 & $\begin{array}{l}\text { Negative control for QS } \\
\text { properties. }\end{array}$ & Gift from Prof. Paul Williams \\
\hline & $\begin{array}{l}\text { luxRluxl' (Photobacterium } \\
\text { fischeri [ATCC }\end{array}$ & \\
\hline Escherichia coli [pSB401] & $\begin{array}{l}\text { 7744]):luxCDABE } \\
\text { (Photorhabdus luminescens } \\
\text { [ATCC 29999]) fusion; } \\
\text { pACYC184-derived, tet }{ }^{R} \text {. } \\
\text { Biosensor that produces } \\
\text { bioluminescence in the presence } \\
\text { of short chain exogenous AHL. }\end{array}$ & {$[24]$} \\
\hline Pandoraea sp. RB-44 & Soil isolate & This study \\
\hline
\end{tabular}

\subsection{Detection of AHL Production Using C. Violaeum CV026 Biosensor}

Preliminary screening of AHL production was done by cross streaking the isolate with $C$. violaeum CV026 on LB agar and incubated for $24 \mathrm{~h}$ at $28{ }^{\circ} \mathrm{C}$. Fomation of purple pigmentation after $24 \mathrm{~h}$ of incubation indicates production of short chain exogenous AHLs from the isolate. E. carotovora GS101 and E. carotovora PNP22 was used as positive and negative controls, respectively. 


\subsection{AHL Extraction}

Pure culture of isolate RB-44 was grown in LB broth buffered to pH 5.5 with $50 \mathrm{mM}$ MOPS and incubated at $28{ }^{\circ} \mathrm{C}$ with shaking for $18 \mathrm{~h}$. The spent supernatant was extracted twice with equal volume of acidified ( $0.1 \% \mathrm{v} / \mathrm{v}$ glacial acetic acid) ethyl acetate as described preciously [25]. AHL extracts was desiccated to complete dryness before further analysis.

\subsection{Bioluminescence Assay Using E. coli [pSB401]}

Bioluminescence expression was determined using Infinite M200 luminometer (Tecan, Männerdorf, Switzerland) in a 96-well microtitre plates as described previously with slight modification [26]. Briefly, overnight culture of biosensor (E. coli [pSB401]) was diluted to an $\mathrm{OD}_{600 \mathrm{~nm}}$ of 0.1 . Then, diluted biosensor cells culture $(250 \mu \mathrm{L})$ was used to resuspend the extracted AHL and added into each well of 96-well microtitre plates. Bioluminescence and optical density $\left(\mathrm{OD}_{495 \mathrm{~nm}}\right)$ were determined at 60 min intervals for $24 \mathrm{~h}$. Bioluminescence expression was expressed as relative light unit per $\mathrm{OD}_{495 \mathrm{~nm}}\left(\mathrm{RLU} / \mathrm{OD}_{495 \mathrm{~nm}}\right)$ against time [26]. Experiments were conducted in triplicate and repeated thrice.

\subsection{Strain Identification Using $16 S$ rDNA Amplification}

The 16S rDNA was PCR amplified using 27F forward primers (5'-AGAGTTTGATCMTGGCTCAG-3'), $515 \mathrm{~F}$ forward primers (5'-GTGCCAGCMGCCGCGGTAA-3') and 1525R reverse primers (5'-AAGGAGGTGWTCCARCC-3') using PCR mix (Promega Kit, Madison, WI, USA) while the genomic DNA was extracted using MasterPure ${ }^{\mathrm{TM}}$ DNA Purification Kit (EPICENTRE Inc., Madison, WI, USA). The PCR amplification was carried out consisting of an initial denaturation of $94{ }^{\circ} \mathrm{C}$ for 3 min, followed by 30 repeated cycles of $94{ }^{\circ} \mathrm{C}$ for $30 \mathrm{~s}$ of denaturing, $60{ }^{\circ} \mathrm{C}$ for $30 \mathrm{~s}$ of annealing and $72{ }^{\circ} \mathrm{C}$ for $1 \mathrm{~min} 30 \mathrm{~s}$ of extension, and a final extension of $72{ }^{\circ} \mathrm{C}$ for $7 \mathrm{~min}$. Product sequence alignment was done using GenBank Blastn database and phylogenetic analysis was done using Molecular Evolutionary Genetic Analysis (MEGA) version 5.0 [27].

\subsection{Matrix-Assisted Laser Desorption Ionization Time-of-Flight Mass Spectrometry (MALDI-TOF MS) Identification}

Sample preparation for MALDI-TOF MS analysis was conducted as described previously [28]. Briefly, fresh culture on LB agar was smeared on MSP 96 target polished steel BC plate and overlaid with $1 \mu \mathrm{L}$ of MALDI matrix (10 mg/mL $\alpha$-cyano-4-hydroxycinnamic acid in 50\% acetonitrile/2.5\% trifluoroacetic acid). The sample was air dried before further analysis in Microflex MALDI-TOF (Bruker Daltonik GmbH, Leipzig, Germany) bench-top mass spectrometer (equipped with UV laser at wavelength $337 \mathrm{~nm}$ ). The built in support software used was Bruker FlexControl software version 3.3 (Build 108) and the spectra were recorded in the linear positive ion mode and analyzed the mass in range of 2 to $20 \mathrm{kDa}$. Each well on the sample plate was measured with laser shots at various random positions and the bacterial MS spectra was analyzed using Bruker MALDI Biotyper Real Time Classification Classification software (Version 3.1, Build 65). The identity of the sample was evaluated based on a dedicated scoring system where the spectra information of the sample was compared to the best match in the Bruker database. If the final score of the spectra value is between 2.3 
to 3.0, the accuracy of the isolate identity is accurate until the level of species; for spectra values between 2.3 to 3.0, the accuracy is secure at the level of genus; for spectra values between 1.7 to 2.0, the accuracy of identity is below the level of genus and for values lower than 1.7, no reliable identity is generated. A dendrogram was then constructed from the standard MALDI Biotyper MSP creation method (Bruker Daltonics, Bremen, Germany) where a phylogenetic tree was generated by similarity scoring of a set of mass spectra illustrating graphical distance values between species constructed from their MALDI-TOF reference spectra.

\subsection{Identification of AHL Profile by High Resolution Tandem Liquid Chromatography Quadrupole Mass Spectrometry (LC-MS/MS)}

An Agilent 1290 Infinity LC system (Agilent Technologies Inc., Santa Clara, CA, USA) was used as the LC delivery system. The column used was the HPLC column C18 column (Agilent Technologies Inc., Santa Clara, CA, USA); $2.1 \mathrm{~mm} \times 50 \mathrm{~mm}, 1.8 \mu \mathrm{m}$ particle sże where the injection volume was set to $2 \mu \mathrm{L}$. LC analysis was carried out at $37^{\circ} \mathrm{C}$ column temperature with an elution of 15 min at constant flow rate of $0.3 \mathrm{~mL} / \mathrm{min}$. Mobile phase A and B were $0.1 \% \mathrm{v} / \mathrm{v}$ formic acid in HPLC grade MilliQ water and $0.1 \% \mathrm{v} / \mathrm{v}$ formic acid in acetonitrile (ACN) respectively. The gradient profiles for the HPLC condition were as follows at (time: mobile phase A: mobile phase B): 0 min: 80:20, 7 min: 50:50, 12 min: 20:80, and 14 min: 80:20.

High Resolution Tandem LC-MS/MS was performed with the Agilent 6490 Triple Quadrupole LC/MS system (Agilent Technologies Inc., Santa Clara, CA, USA). The ion source used is in electrospray ionization (ESI) positive mode.The probe capillary voltage was set at $3 \mathrm{kV}$, nebulizer pressure at $20 \mathrm{psi}$, sheath gas at $11 \mathrm{~mL} / \mathrm{h}$, desolvation temperature at $200{ }^{\circ} \mathrm{C}$, collision energy at $5 \mathrm{eV}$ and fragmentation at $380 \mathrm{eV}$. For detection of AHLs, precursor ion scan mode is used where the product ion $\mathrm{m} / \mathrm{z}$ was set as 102 indicating the $[\mathrm{M}+\mathrm{H}]^{+}$ion of the core lactone ring moiety. The $\mathrm{m} / \mathrm{z}$ value range of the precursor ions was then set from 150 to 400. Agilent Mass Hunter software was used for the MS data analysis by comparison of extracted ion (EI) mass spectra and retention index with synthetic AHL compounds.

\section{Results and Discussion}

\subsection{Sampling and Screening for AHL Producing Bacteria}

The sample site was previously a landfill site for domestic household waste materials and had ceased operation since 7 years ago. The temperature of the soil surface was $33^{\circ} \mathrm{C}$ in daytime and $\mathrm{pH}$ reading of value 7 was documented on the day when sampling was done. The soil sample was collected in the middle of the ex-landfill site.

A wide variety of bacterial biosensors have been constructed for screening of AHL production [23,24,29]. Most of these biosensors function with a mutated AHL synthase but with a functional AHL receptor remains intact that could respond to the presence of exogenous AHLs by the activation of a reporter gene such as lacZ or lux operon. In this study, C. violaceum CV026 biosensor was used for routine screening as it is a rapid and accurate preliminary QS screening [23]. 
Subsequently, further analysis was conducted with bioluminescence assay in lux-based E. coli biosensors followed by high resolution analytical instrument such as mass spectrometry analysis.

Figure 1. AHL screening of strain RB-44 with C. violaeum CV026. E. carotovora GS101 and E. carotovora PNP22 refer to the postive and negative controls, respectively.

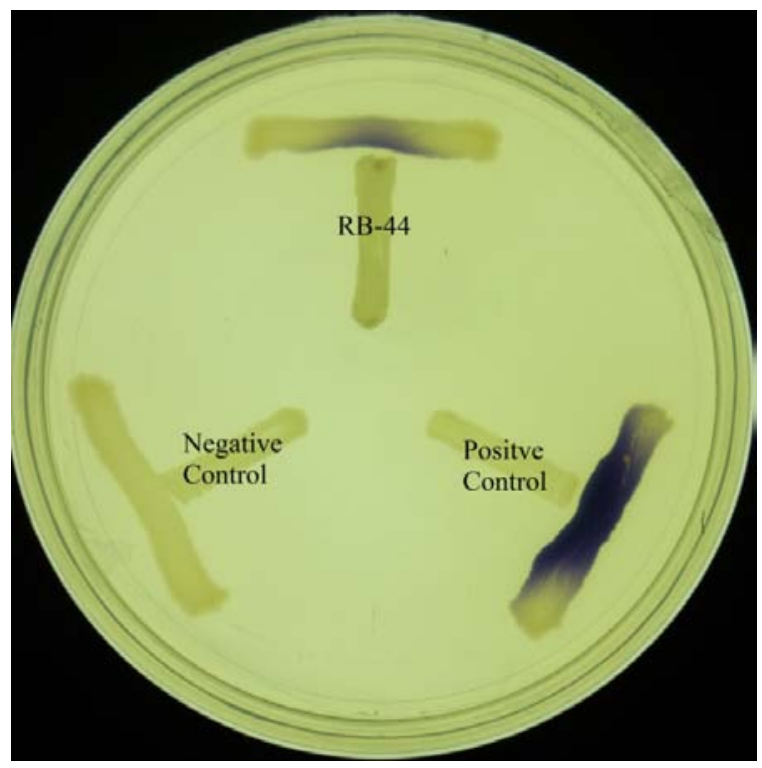

About 60 strains were isolated after four cycles of enrichment in KGm medium with 3-oxo-C6-HSL as the sole carbon sources. Preliminary screening for AHL production using C. violaeum CV026 biosensor (Figure 1) suggested strain RB-44 showed QS activity and moderate quorum quenching actitivity for 3-oxo-C6-HSL (data not shown). Strain RB-44 was selected for further QS analysis since it triggered C. violaeum CV026 biosensor purple pigement formation and subsequently reconfirmed its AHL production by using E. coli [pSB401] bioluminescence assay (Figure 2).

Figure 2. Bioluminescence assay using E. coli [pSB401] biosensor. Graph was plotted as $\mathrm{RLU} \mathrm{OD}_{495 \mathrm{~nm}}$ against time. AHL production by strain RB-44 (circle) is confirmed the increased value of RLU/OD $495 \mathrm{~nm}$ and observed over 24 h. Negative control (square) was included with extracted AHL in blank LB. Each point represents the mean and error of results from independent triplicate cultures.

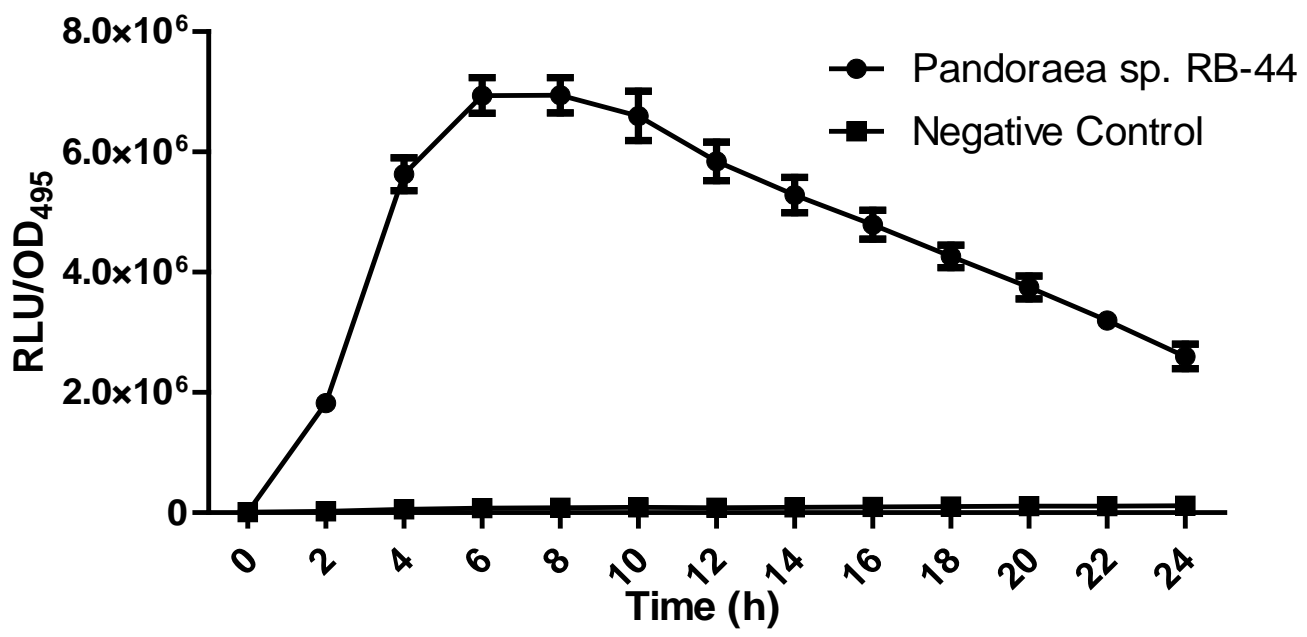




\subsection{Strain Identification and MALDI-TOF Score-Oriented Dendrogram}

There are many reports in clinical laboratories that conventional methods are not able to identify Pandoraea spp. even when using a widely accepted phenotypic method [30,31]. There is even a quality assurance trial of CF microbiology which Pandoraea pnomenusa strain was misclassified or not even detected by many laboratories that reinforced this notion [32]. Thus, MALDI-TOF mass spectrometry represents a more reliable method and high throughput for the classification and identification of microorganisms with a shorter turn over time [33,34]. Unlike other cystic fibrosis-causing agents, no biofilm formation is observed from overnight culture of strain RB-44.

MALDI-TOF MS is a rapid and yet promising means of accurate identification of microorganisms [35], especially for the Pandoraea genus that is often misidentified as Burkholderia spp. or Ralstonia spp. [31]. In this study, MALDI-TOF MS result accurately showed that the closest match of the RB-44 isolate is Pandoraea sp.with a best match score value of 2.415 and $P$. pnomenusa as the second best match (score value of 2.382) in the MALDI-TOF Biotyper Real Time Classification software. MALDI Biotyper MSP software also showed a closer match to Pandoraea sp. (Figure 3).

Figure 3. Score-orientated dendrogram that shows classification of Pandoraea sp. RB-44. Bacterial strain RB-44 is clustered hierarchically based on the protein mass spectra patterns.

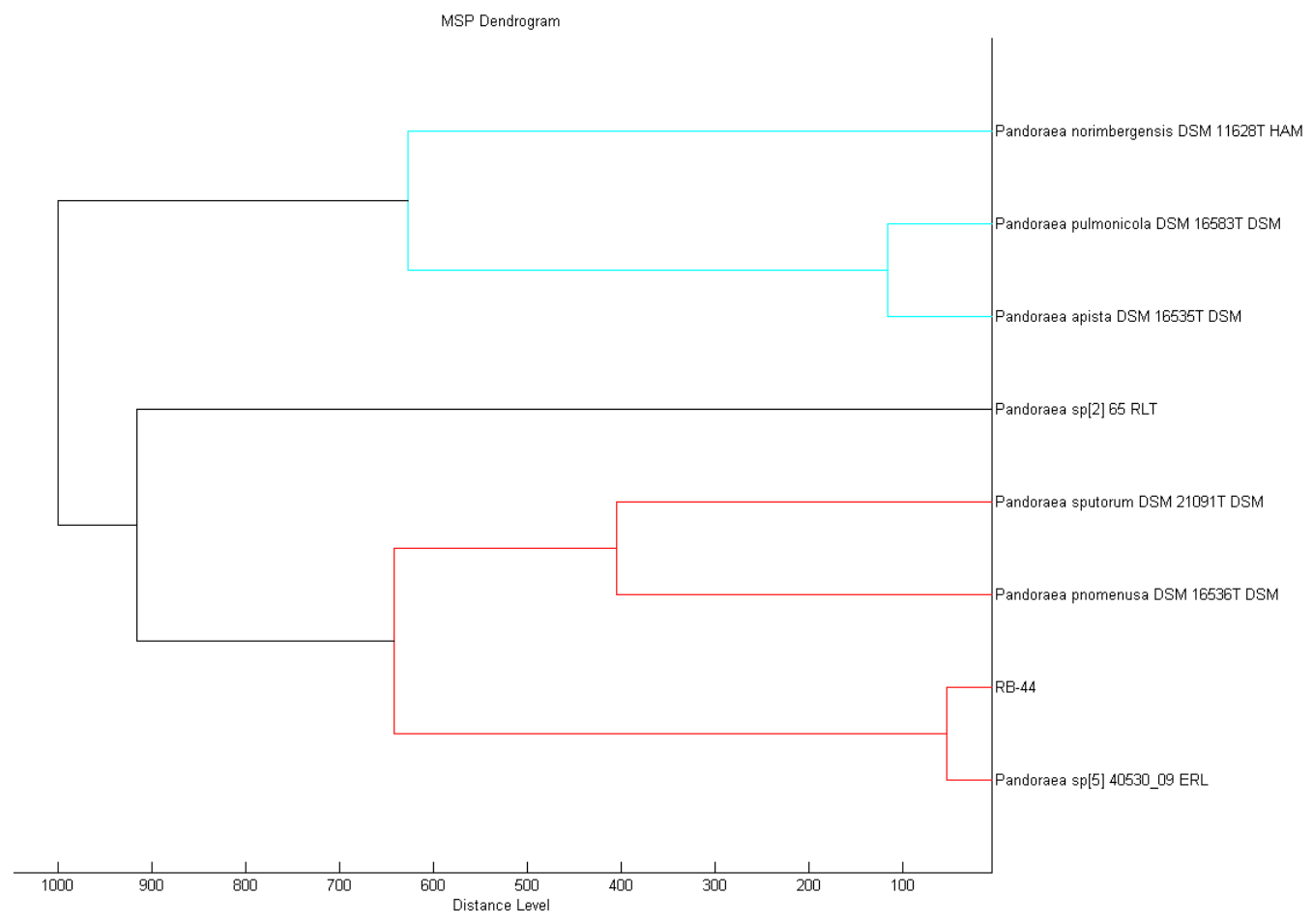

The identity of strain RB-44 is also confirmed by 16S rDNA sequencing showing that it clusters within the Pandoraea genus. The sequence was subsequently deposited into GenBank with the accession number KF648559. A phylogenetic tree was then constructed using Mega 5 software to align it with other rDNA sequences obtained from GenBank. Based on the phylogenetic tree obtained from MALDI-TOF and 16S rDNA sequencing (Figure 4), it appears that strain RB-44 could represent a new species of Pandoraea genus. However, more research should be to be conducted to confirm this finding. 
Figure 4. The evolutionary history was inferred using the Neighbor-Joining method [36]. The optimal tree with the sum of branch length $=0.08118917$ is shown. The percentage of replicate trees in which the associated taxa clustered together in the bootstrap test $(1,000$ replicates) are shown next to the branches [37]. The tree is drawn to scale, with branch lengths in the same units as those of the evolutionary distances used to infer the phylogenetic tree. The evolutionary distances were computed using the Maximum Composite Likelihood method [38] and are in the units of the number of base substitutions per site. The analysis involved 13 nucleotide sequences. Codon positions included were 1st+2nd+3rd+Noncoding. All positions containing gaps and missing data were eliminated. There were a total of 1388 positions in the final dataset. Evolutionary analyses were conducted in MEGA 5.0 [27].

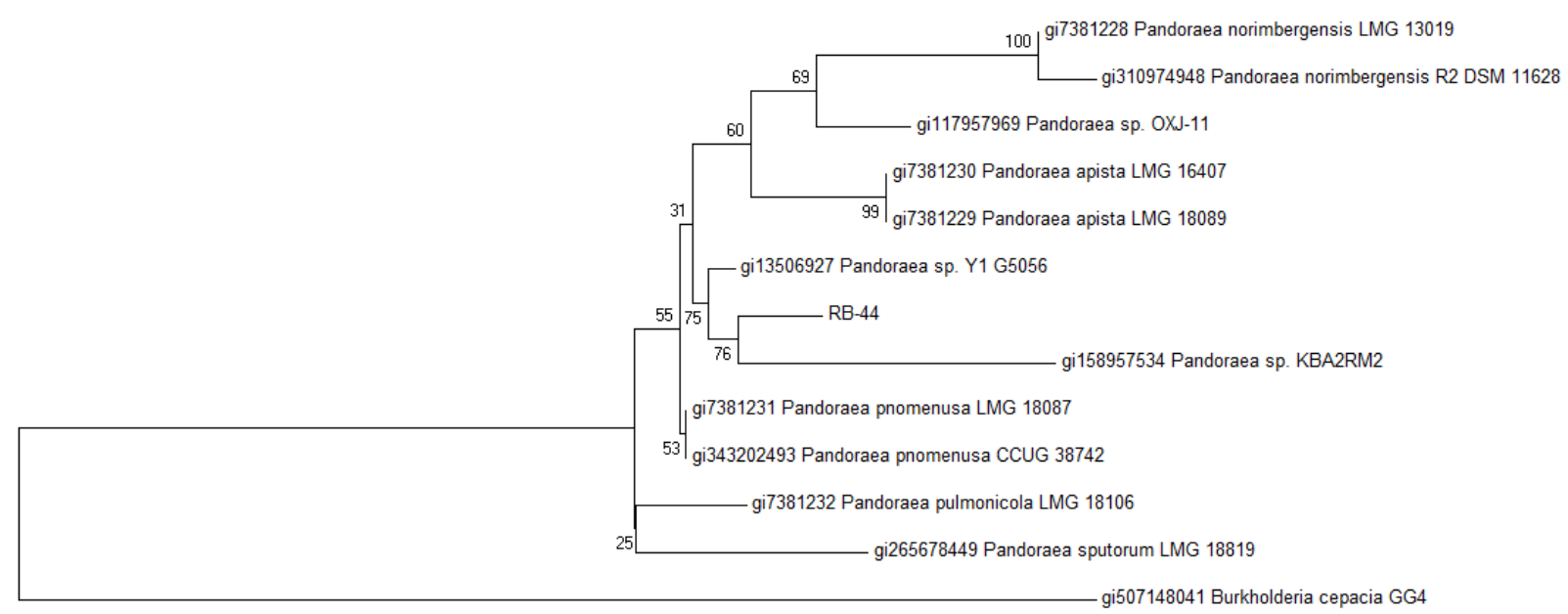

gi507148041 Burkholderia cepacia GG4

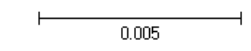

3.3. Identification of AHL Profile by High Resolution Tandem Liquid Chromatography Quadrupole Mass Spectrometry (MS/MS)

Many cystic fibrosis-causing agents such as Pseudomonas aeruginosa and Burkholderia spp. have been reported to possess QS properties as a mechanism to regulate virulence. As an opportunistic pathogen with an unclear role, Pandoraea spp. have repeatedly been isolated and reported to be co-colonizing species in cystic fibrosis patients [5,31,34]. Thus, identification of the Pandoraea spp. AHL profile is the first step to understand this potential opportunistic pathogen. The spent culture supernatants of Pandoraea sp. RB-44 was analyzed using an Agilent 6490 Triple Quadrupole LC-MS/MS system. Mass spectrometry analysis of the supernatant of Pandoraea sp. RB-44 confirmed the presence of $\mathrm{N}$-octanoylhomoserine lactone (C8-HSL) ( $\mathrm{m} / \mathrm{z}$ value of 228.200) (Figure 5). 
Figure 5. Mass spectrometry analysis of C8-HSL from an AHL standard (a) showed the $\mathrm{m} / \mathrm{z}$ value 228.100; retention time: $4.633 \mathrm{~min}$; abundance: 115,382.02 and abundance \%: 100, while C8-HSL produced by Pandoraea sp. RB-44 (b) showed m/z value: 228.200; retention time: 4.633 min; abundance: 2,459.14 and abundance \%: 100.

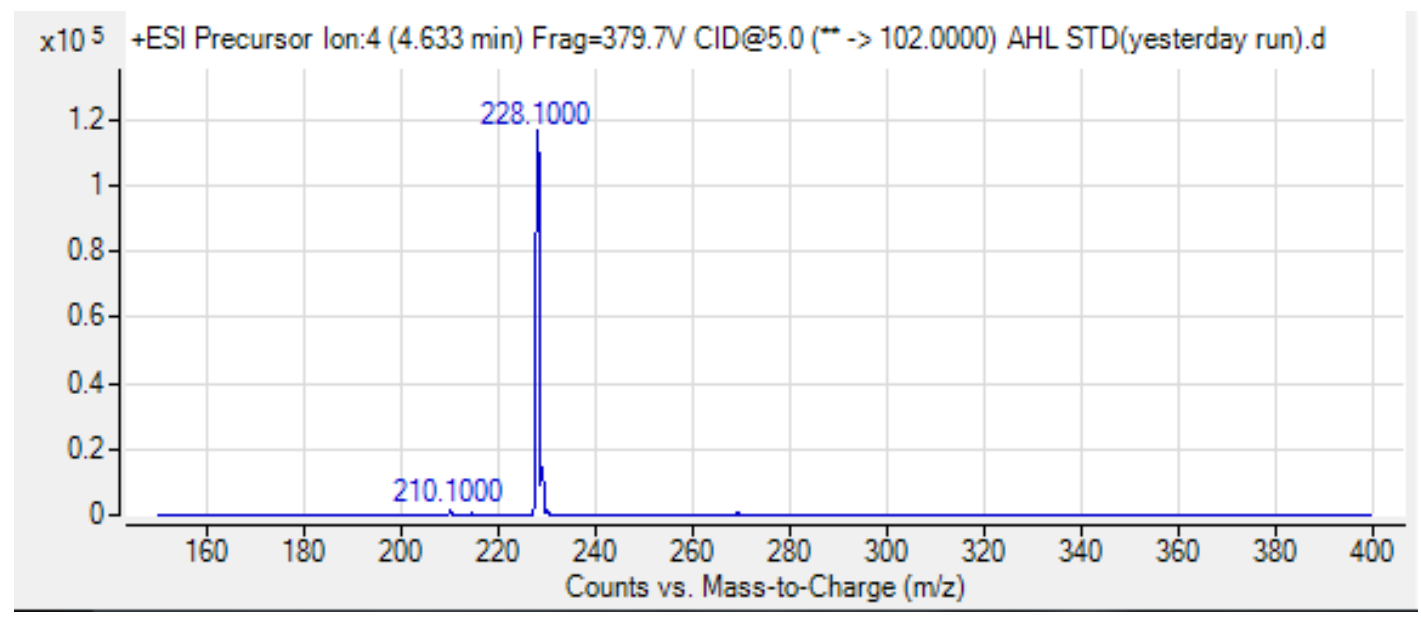

(a)

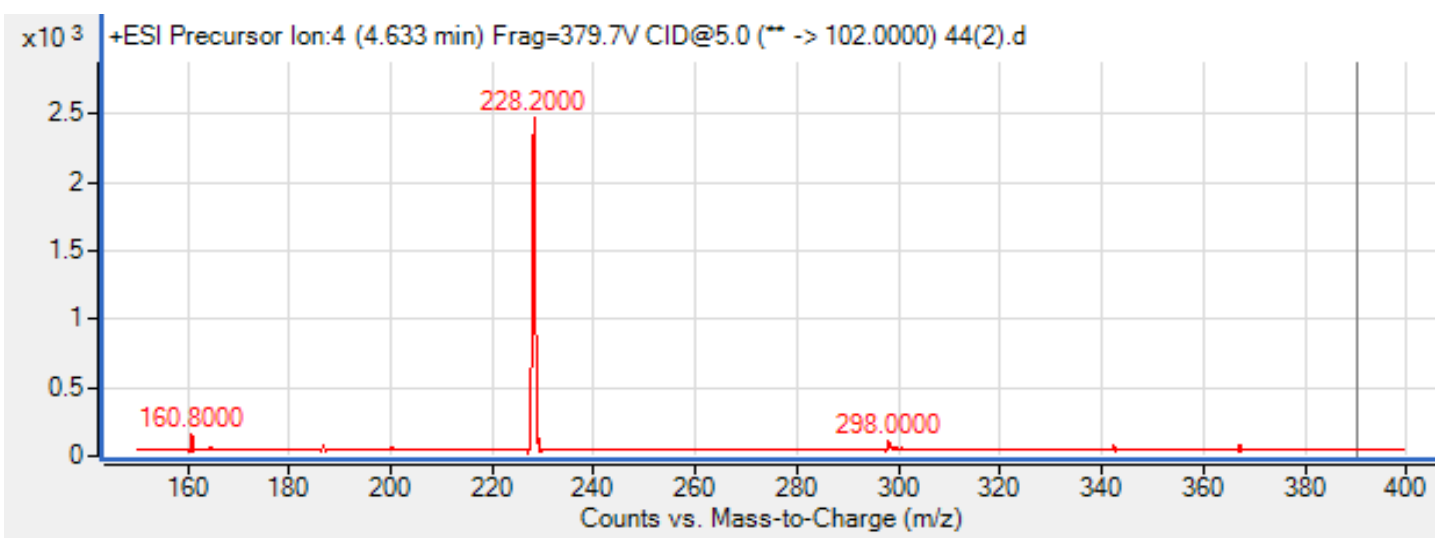

(b)

\section{Conclusions/Outlook}

We report here the AHL profile of Pandoraea sp. RB-44 isolated from an ex-landfill site. A short chain AHL, namely C8-HSL, was detected in the spent culture supernatant of Pandoraea sp. RB-44. To the best of our knowledge, this is the first documentation of AHL production by a member of the Pandoraea. Further investigations are being carried out to confirm the QS gene of Pandoraea sp. RB-44.

\section{Acknowledgments}

This research was supported by the University of Malaya HIR Grant (A-000001-50001) to Kok-Gan Chan which is gratefully acknowledged. We thank Paul Williams (University of Nottingham, UK) for providing us the biosensor.

\section{Conflicts of Interest}

The authors declare no conflict of interest. 


\section{References}

1. Coenye, T.; Liu, L.; Vandamme, P.; LiPuma, J.J. Identification of Pandoraea species by $16 \mathrm{~S}$ ribosomal DNA-based PCR assays. J. Clin. Microbiol. 2001, 39, 4452-4455.

2. Caraher, E.; Collins, J.; Herbert, G.; Murphy, P.G.; Gallagher, C.G.; Crowe, M.J.; Callaghan, M.; McClean, S. Evaluation of in vitro virulence characteristics of the genus Pandoraea in lung epithelial cells. J. Med. Microbiol. 2008, 57, 15-20.

3. Aravena-Roman, M. Cellular fatty acid-deficient Pandoraea isolated from a patient with cystic fibrosis. J. Med. Microbiol. 2008, 57, 252.

4. Coenye, T.; Falsen, E.; Hoste, B.; Ohlen, M.; Goris, J.; Govan, J.R.; Gillis, M.; Vandamme, P. Description of Pandoraea gen. nov. with Pandoraea apista sp. nov., Pandoraea pulmonicola sp. nov., Pandoraea pnomenusa sp. nov., Pandoraea sputorum sp. nov. and Pandoraea norimbergensis comb. nov. Int. J. Syst. Evol. Microbiol. 2000, 2, 887-899.

5. Atkinson, R.M.; Lipuma, J.J.; Rosenbluth, D.B.; Dunne, W.M., Jr. Chronic colonization with Pandoraea apista in cystic fibrosis patients determined by repetitive-element-sequence PCR. J. Clin. Microbiol. 2006, 44, 833-836.

6. Daneshvar, M.I.; Hollis, D.G.; Steigerwalt, A.G.; Whitney, A.M.; Spangler, L.; Douglas, M.P.; Jordan, J.G.; MacGregor, J.P.; Hill, B.C.; Tenover, F.C.; et al. Assignment of CDC weak oxidizer group 2 (WO-2) to the genus Pandoraea and characterization of three new Pandoraea genomospecies. J. Clin. Microbiol. 2001, 39, 1819-1826.

7. Stryjewski, M.E.; LiPuma, J.J.; Messier, R.H., Jr.; Reller, L.B.; Alexander, B.D. Sepsis, multiple organ failure, and death due to Pandoraea pnomenusa infection after lung transplantation. J. Clin. Microbiol. 2003, 41, 2255-2257.

8. Jorgensen, I.M.; Johansen, H.K.; Frederiksen, B.; Pressler, T.; Hansen, A.; Vandamme, P.; Hoiby, N.; Koch, C. Epidemic spread of Pandoraea apista, a new pathogen causing severe lung disease in cystic fibrosis patients. Pediatr. Pulmonol. 2003, 36, 439-446.

9. Rani, A.; Porwal, S.; Sharma, R.; Kapley, A.; Purohit, H.J.; Kalia, V.C. Assessment of microbial diversity in effluent treatment plants by culture dependent and culture independent approaches. Bioresour. Technol. 2008, 99, 7098-7107.

10. Dhindwal, S.; Patil, D.N.; Mohammadi, M.; Sylvestre, M.; Tomar, S.; Kumar, P. Biochemical studies and ligand-bound structures of biphenyl dehydrogenase from Pandoraea pnomenusa strain B-356 reveal a basis for broad specificity of the enzyme. J. Biol. Chem. 2011, 286, 37011-37022.

11. Anandham, R.; Indiragandhi, P.; Madhaiyan, M.; Ryu, K.Y.; Jee, H.J.; Sa, T.M. Chemolithoautotrophic oxidation of thiosulfate and phylogenetic distribution of sulfur oxidation gene (soxB) in rhizobacteria isolated from crop plants. Res. Microbiol. 2008, 159, 579-589.

12. Shi, Y.; Chai, L.; Tang, C.; Yang, Z.; Zheng, Y.; Chen, Y.; Jing, Q. Biochemical investigation of kraft lignin degradation by Pandoraea sp. B-6 isolated from bamboo slips. Bioproc. Biosyst. Eng. 2013, doi:10.1007/s00449-013-0972-9.

13. Fuqua, C.; Winans, S.C.; Greenberg, E.P. Census and consensus in bacterial ecosystems: The LuxR-LuxI family of quorum-sensing transcriptional regulators. Annu. Rev. Microbiol. 1996, 50, 727-751. 
14. Fuqua, C.; Greenberg, E.P. Self perception in bacteria: Quorum sensing with acylated homoserine lactones. Curr. Opin. Microbiol. 1998, 1, 183-189.

15. Williams, P. Quorum sensing, communication and cross-kingdom signalling in the bacterial world. Microbiology 2007, 153, 3923-3938.

16. Salmond, G.P.; Bycroft, B.W.; Stewart, G.S.; Williams, P. The bacterial “enigma”: Cracking the code of cell-cell communication. Mol. Microbiol. 1995, 16, 615-624.

17. Hardman, A.M.; Stewart, G.S.; Williams, P. Quorum sensing and the cell-cell communication dependent regulation of gene expression in pathogenic and non-pathogenic bacteria. Antonie. Van Leeuwenhoek 1998, 74, 199-210.

18. Swift, S.; Throup, J.P.; Williams, P.; Salmond, G.P.; Stewart, G.S. Quorum sensing: A population-density component in the determination of bacterial phenotype. Trends Biochem. Sci. 1996, 21, 214-219.

19. Swift, S.; Downie, J.A.; Whitehead, N.A.; Barnard, A.M.; Salmond, G.P.; Williams, P. Quorum sensing as a population-density-dependent determinant of bacterial physiology. Adv. Microb. Physiol. 2001, 45, 199-270.

20. Hong, K.-W.; Koh, C.-L.; Sam, C.-K.; Yin, W.-F.; Chan, K.G. Quorum quenching revisited-Fromsignal decays to signalling confusion. Sensors 2012, 12, 4661-4696.

21. Wong, C.S.; Yin, W.F.; Choo, Y.M.; Sam, C.K.; Koh, C.L.; Chan, K.G. Coexistence of quorum-quenching and quorum-sensing in tropical marine Pseudomonas aeruginosa strain MW3A. World J. Microbiol. Biotechnol. 2012, 28, 453-461.

22. Chan, K.G.; Yin, W.F.; Sam, C.K.; Koh, C.L. A novel medium for the isolation of $\mathrm{N}$-acylhomoserine lactone-degrading bacteria. J. Ind. Microbiol. Biotechnol. 2009, 36, 247-251.

23. McClean, K.H.; Winson, M.K.; Fish, L.; Taylor, A.; Chhabra, S.R.; Camara, M.; Daykin, M.; Lamb, J.H.; Swift, S.; Bycroft, B.W.; Stewart, G.S.; Williams, P. Quorum sensing and Chromobacterium violaceum: Exploitation of violacein production and inhibition for the detection of $N$-acylhomoserine lactones. Microbiology 1997, 143, 3703-3711.

24. Winson, M.K.; Swift, S.; Fish, L.; Throup, J.P.; Jorgensen, F.; Chhabra, S.R.; Bycroft, B.W.; Williams, P.; Stewart, G.S. Construction and analysis of luxCDABE-based plasmid sensors for investigating $N$-acyl homoserine lactone-mediated quorum sensing. FEMS Microbiol. Lett. 1998, 163, 185-192.

25. Ortori, C.A.; Dubern, J.F.; Chhabra, S.R.; Camara, M.; Hardie, K.; Williams, P.; Barrett, D.A. Simultaneous quantitative profiling of N-acyl-L-homoserine lactone and 2-alkyl-4(1H)-quinolone families of quorum-sensing signaling molecules using LC-MS/MS. Anal. Bioanal. Chem. 2011, 399, 839-850.

26. Tan, L.Y.; Yin, W.F.; Chan, K.G. Silencing quorum sensing through extracts of Melicope lunu-ankenda. Sensors 2012, 12, 4339-4351.

27. Tamura, K.; Peterson, D.; Peterson, N.; Stecher, G.; Nei, M.; Kumar, S. MEGA5: Molecular evolutionary genetics analysis using maximum likelihood, evolutionary distance, and maximum parsimony methods. Mol. Biol. Evol. 2011, 28, 2731-2739. 
28. Mellmann, A.; Cloud, J.; Maier, T.; Keckevoet, U.; Ramminger, I.; Iwen, P.; Dunn, J.; Hall, G.; Wilson, D.; Lasala, P.; et al. Evaluation of matrix-assisted laser desorption ionization-time-offlight mass spectrometry in comparison to 16S rRNA gene sequencing for species identification of nonfermenting bacteria. J. Clin. Microbiol. 2008, 46, 1946-1954.

29. Shaw, P.D.; Ping, G.; Daly, S.L.; Cha, C.; Cronan, J.E., Jr.; Rinehart, K.L.; Farrand, S.K. Detecting and characterizing $N$-acyl-homoserine lactone signal molecules by thin-layer chromatography. Proc. Natl. Acad. Sci. USA 1997, 94, 6036-6041.

30. Brisse, S.; Stefani, S.; Verhoef, J.; Van Belkum, A.; Vandamme, P.; Goessens, W. Comparative evaluation of the BD Phoenix and VITEK 2 automated instruments for identification of isolates of the Burkholderia. cepacia complex. J. Clin. Microbiol. 2002, 40, 1743-1748.

31. Fernandez-Olmos, A.; Morosini, M.I.; Lamas, A.; Garcia-Castillo, M.; Garcia-Garcia, L.; Canton, R.; Maiz, L. Clinical and microbiological features of a cystic fibrosis patient chronically colonized with Pandoraea sputorum identified by combining 16S rRNA sequencing and matrix-assisted laser desorption ionization-time of flight mass spectrometry. J. Clin. Microbiol. 2012, 50, 1096-1098.

32. Hogardt, M.; Ulrich, J.; Riehn-Kopp, H.; Tummler, B. Euro Care CF quality assessment of diagnostic microbiology of cystic fibrosis isolates. J. Clin. Microbiol. 2009, 47, 3435-3438.

33. vanBaar, B.L. Characterisation of bacteria by matrix-assisted laser desorption/ionisation and electrospray mass spectrometry. FEMS Microbiol. Rev. 2000, 24, 193-219.

34. Coenye, T.; Goris, J.; Spilker, T.; Vandamme, P.; LiPuma, J.J. Characterization of unusual bacteria isolated from respiratory secretions of cystic fibrosis patients and description of Inquilinus limosusgen. nov., sp. nov. J. Clin. Microbiol. 2002, 40, 2062-2069.

35. Chen, J.W.; Koh, C.L.; Sam, C.K.; Yin, W.F.; Chan, K.G. Short chain n-acyl homoserine lactone production by soil isolate Burkholderia sp. strain A9. Sensors 2013, 13, 13217-13227.

36. Saitou, N.; Nei, M. The neighbor-joining method: A new method for reconstructing phylogenetic trees. Mol. Biol. Evol. 1987, 4, 406-425.

37. Felsenstein J. Confidence limits on phylogenies: An approach using the bootstrap. Evolution 1985, 39, 783-791.

38. Tamura, K.; Nei, M.; Kumar, S. Prospects for inferring very large phylogenies by using the neighbor-joining method. Proc. Natl. Acad. Sci. USA 2004, 101, 11030-11035.

(C) 2013 by the authors; licensee MDPI, Basel, Switzerland. This article is an open access article distributed under the terms and conditions of the Creative Commons Attribution license (http://creativecommons.org/licenses/by/3.0/). 